\title{
THE APPLICATION OF DIFFUSION MODELS TO AN AGGREGATED SOIL ${ }^{1}$
}

\section{RAPPOLDT ${ }^{2}$}

In models on diffusive transport it is necessary to make use of a simplifying description of the geometry of an aggregated soil. It is proposed to use a (hypothetical) model soil consisting of spherical, cylindrical, or flat aggregates. The model aggregates are of different sizes, and each size class occupies a certain fraction of the total volume. These volume fractions can be calculated in such a way that diffusive transports take place in approximately the same way in soil and model soil.

The method is operational. The quantity to be measured is the shortest distance from a randomly chosen point to the interaggregate space. The desired volume fractions can be derived from the probability distribution of this distance. The procedure does not require the existence of individual aggregates and may be applied, for instance, to a cracked clay soil.

The use of a simple model soil is based on the shape independence of diffusion processes. That shape independence has been verified by means of theoretical calculations for both a single aggregate and an aggregated soil.

In the literature detailed studies can be found on diffusion processes in soil aggregates. Smith (1977) used a model for oxygen diffusion and soil respiration to describe anaerobiosis, and Leffelaar (1987) studied water flow and gas diffusion in relation to denitrification. In general, a diffusion model will be based on a differential equation describing the process taking place. The equation contains a term for diffusive transport, and there may be source and sink terms describing chemical reactions.

The application of a model to an aggregated soil means that a differential equation is solved for the whole soil. That requires a simplifying description of the soil geometry. In this paper appropriate simplifications are studied for a sin-

${ }^{1}$ This research was made possible by a grant from the Netherlands Integrated Soil Research Program.

${ }^{2}$ Department of Theoretical Production Ecology, Agricultural University, P.O. Box 430, 6700 AK Wageningen, The Netherlands.

Received 2 Nov, 1988; revised 4 Dec. 1989. gle aggregate with an irregular shape and for an aggregated soil.

For a single aggregate there is an approximate method available that is commonly used by process engineers. The real aggregate is replaced by a simpler one for which all calculations are carried out. The simple model aggregate should only have the same surface-to-volume ratio as the real aggregate. In fact, this ratio $S / V$ is the only geometric property of the aggregate used.

An aggregated soil consists of a porous medium and of macropores. The porous medium may be present in the form of separate aggregates, but that is not necessarily the case. It may also consist of structures with a complicated shape, in which no separate aggregates can be distinguished (Fig. 1). These structures may have very different sizes, and their surface may be rough. The porous medium will be denoted as the intra-aggregate space and the macropores as the inter-aggregate space. A cracked clayey soil, for instance, consists of (interconnected) clods and cracks, forming the intra- and interaggregate spaces, respectively.

The proposed method for applying a diffusion model to an aggregated soil is a generalization of the method for a single aggregate. This generalization is the main subject of this paper. The geometry of a soil is characterized by means of a function closely related to an $S / V$ ratio. This function can be derived from images of the soil structure. Hence the method is operational and can be used, in principle, to combine image analysis with diffusion models.

\section{METHOD FOR A SINGLE AGGREGATE}

\section{An equivalent aggregate}

A single aggregate with an arbitrary shape is considered. It has an outer surface $S$ and a volume $V$. It is shown in this section that the result of a diffusion process is largely determined by the surface-to-volume ratio $S / V$. Aggregate shape is of minor importance, and the actual aggregate shape may be replaced by the simpler shape of an equivalent aggregate. An equivalent aggregate is a hypothetical sphere, cylinder, or plane sheet with the same $S / V$ ratio as the real aggregate. 


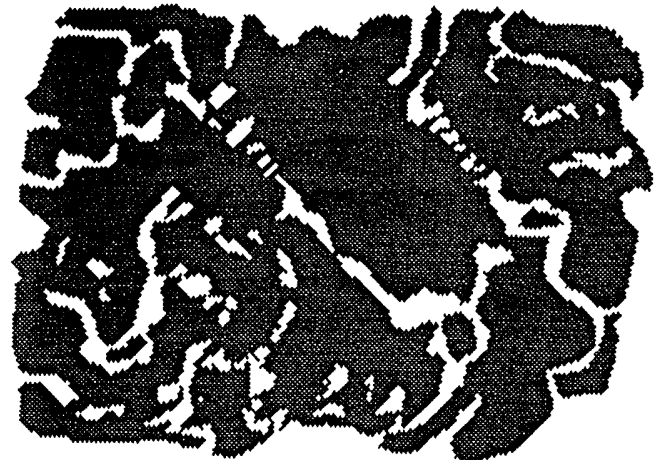

FIG. 1. An aggregated soil may consist of interconnected clods forming a complicated structure. The drawing shows a hypothetical intersection.

A sphere is fully described by its radius $R$. Its surface-to-volume ratio is $3 / R$, and the radius of the equivalent sphere belonging to a certain $S / V$ ratio becomes $3 \mathrm{~V} / \mathrm{S}$. The cylinders considered in this paper are long, and any influence of the "top" and "bottom" surfaces is neglected. An equivalent cylinder is therefore described by its radius, which is calculated as $2 \mathrm{~V} / \mathrm{S}$. Finally, plane sheets are large and flat; "end" effects are neglected, and a plane sheet is fully described by its thickness. If the plane sheet radius is defined as half its thickness, the radius of an equivalent plane sheet is $1 \mathrm{~V} / \mathrm{S}$.

The use of an equivalent aggregate in model calculations is justified only when aggregate shape has a minor influence on the results. That is verified below for four diffusion models by comparing model results for a sphere, a cylinder, and a plane sheet with the same $S / V$ ratio. The four diffusion models have been taken from the literature. Therefore, only a brief description of each process is given. The results are presented in the form of graphs. These graphs facilitate an assessment of the accuracy of the approximate methods without knowledge of mathematical details of the diffusion models.

\section{Soil respiration as a zero-order absorption process}

The model used originates from Greenwood (1961) and Currie (1961) and has been described also by Smith (1977) and Arah and Smith (1989). In this paper a slightly simplified version is used, not taking into account soil porosity and the solubility constant of oxygen. This simpli- fication is justified in the Discussion of results for a single aggregate.

Soil respiration is described as a zero-order absorption process, implying that in aerobe parts of the soil the respiration $Q$ is independent of the local oxygen concentration. An anaerobe zone, in which no respiration takes place, may occur. The differential equation describing the steady state for a spherical aggregate is

$\frac{\mathrm{d}}{\mathrm{d} r}\left[-D 4 \pi r^{2} \frac{\mathrm{d} c(r)}{\mathrm{d} r}\right]=-Q 4 \pi r^{2}, \quad r_{0} \leq r \leq R$

with boundary conditions

$c(r)=0, \mathrm{~d} c(r) / \mathrm{d} r=0$

for $r=r_{0}$ and $c(R)=C$

Here $c\left[\mathrm{~mol} \mathrm{~m}^{-3}\right]$ is the oxygen concentration, $r[\mathrm{~m}]$ the distance from the center (coordinate), $r_{0}[\mathrm{~m}]$ the unknown radius of the anaerobe zone, $R[\mathrm{~m}]$ the sphere radius, $C\left[\mathrm{~mol} \mathrm{~m}^{-3}\right]$ the constant oxygen concentration outside the aggregate, $Q\left[\mathrm{~mol} \mathrm{~m} \mathrm{~m}^{-3} \mathrm{~s}^{-1}\right]$ the respiration rate, and $D\left[\mathrm{~m}^{2} \mathrm{~s}^{-1}\right]$ the diffusion coefficient.

The group $D C / Q$ is a constant and plays a special role in the solution of Eq. (1). $D C / Q$ has the dimension of a length squared, and its square root is defined as the diffusion distance $d$

$$
d \equiv \sqrt{D C / Q}
$$

The diffusion distance is a characteristic length belonging to the process taking place. Its definition is independent of aggregate geometry. The size of $d$ gives an indication of the distance over which oxygen will enter into an aggregate (of arbitrary shape). When $d$ is much smaller than aggregate size, the diffusive transport will be too small to maintain aerobe conditions throughout the aggregate. This leads to a steadystate anaerobe volume.

Equation (1) can be solved using the first two boundary conditions. The third condition is then used to find the radius of the anaerobe zone $r_{0}$ and leads to

$$
\begin{cases}R^{2}-3 r_{0}{ }^{2}+\frac{2 r_{0}{ }^{3}}{R}=6 d^{2}, & d \leq R / \sqrt{6} \\ r_{0}=0, & d>-R / \sqrt{6}\end{cases}
$$

Equation (2) shows that $r_{0}$ is a function of the sphere radius $R$ and the diffusion distance $d$. 
Writing this as $r_{0}(R, d)$, the anaerobe volume fraction becomes

$$
\varphi_{s}(R, d)=\left(\frac{r_{0}(R, d)}{R}\right)^{3}
$$

By dividing Eq. (2) by $R^{2}$, it may be seen that $r_{0} / R$ and, consequently, $\varphi_{s}$ are a function of the ratio $d / R$ only. When the sphere radius $R$ is replaced then by $3 V / S$, the anaerobe volume fraction becomes a function of $d S / V$, the dimensionless product of the diffusion distance and the surface-to-volume ratio of the sphere. Note that the subscript $s$ refers to a sphere.

For a cylinder, analogous equations can be derived. The radius $r_{0}$ of the anaerobe zone in a cylinder is found by solving (numerically)

$$
\begin{cases}R^{2}+r_{0}{ }^{2}+r_{0}{ }^{2} \ln \left(\frac{r_{0}}{R}\right)^{2}=4 d^{2}, & d \leq R / 2 \\ r_{0}=0, & \mathrm{~d}>\mathrm{R} / 2\end{cases}
$$

And the anaerobe volume fraction $\varphi_{c}(R, d)$ for a cylinder is

$$
\varphi_{c}(R, d)=\left(\frac{r_{0}(R, d)}{R}\right)^{2}
$$

Again replacing $R$ by $2 V / S$, the anaerobe volume fraction becomes a function of $d S / V$.

For a plane sheet the equations are simple. For the radius, $r_{0}$ of the anaerobe zone holds

$$
\begin{cases}r_{0}=R-\sqrt{2} d, & d \leq R / \sqrt{2} \\ r_{0}=0, & d>R / \sqrt{2}\end{cases}
$$

The anerobe volume fraction $\varphi_{p}(R, d)$ of a plane sheet is equal to the ratio $r_{0} / R$. Replacing $R$ by $V / S, \varphi_{p}$ also becomes a function of $d S / V$.

For a spherical, cylindrical, and flat aggregate shape, Fig. 2a shows graphs of the anaerobe volume fraction $\varphi$ as function of $d S / V$. This result will be discussed after descriptions of the other diffusion models are presented.

\section{A first-order absorption process}

The absorption considered in this section is proportional to the concentration at each point. A gas or solute is absorbed, for instance, as the result of a first-order reaction taking place, or the oxygen use of organisms is assumed to be proportional to the oxygen concentration. For a spherical aggregate shape the steady state of the process is described by

$$
\begin{aligned}
\frac{\mathrm{d}}{\mathrm{d} r}\left[-D 4 \pi r^{2} \frac{\mathrm{d} c(r)}{\mathrm{d} r}\right] & \\
& =-A 4 \pi r^{2} c(r), \quad 0 \leq r \leq R
\end{aligned}
$$

with boundary conditions

$$
c(R)=C \text { and } c(0) \text { finite }
$$

Here $A\left[s^{-1}\right]$ is the constant ratio between absorption and concentration.

The solution of Eq. (5) is a concentration profile $c(r)$. At the outer surface of the sphere, for $r=R$, the concentration is equal to $C$. For smaller values of $r$ the steady-state concentration is lower but always larger than zero.

The characteristic length $\lambda$ appearing in the solutions for a sphere, cylinder, and plane sheet is $\sqrt{D / A}$. If $\lambda$ is a few times the aggregate radius, the concentration $c(r)$ approaches the outer concentration everywhere. The absorption (in mol $\mathrm{s}^{-1}$ ) of the complete aggregate approaches then the maximum value $A V C$. The actual absorption divided by this maximum is defined as the effectiveness factor $\eta$.

For spheres, cylinders, and plane sheets $\eta$ is known as a function of the radius $R$ and the length $\lambda$. For spheres, Bird et al. (1960 section 17.6) give a complete derivation of

$$
\eta_{s}(R, \lambda)=3\left(\frac{\lambda}{R}\right)^{2}[(R / \lambda) \operatorname{coth}(R / \lambda)-1]
$$

For cylinders, the solution is (Bird et al. 1960 problem 17.M)

$$
\eta_{c}(R, \lambda)=2\left(\frac{\lambda}{R}\right) \frac{I_{1}(R / \lambda)}{I_{0}(R / \lambda)}
$$

Here $I_{0}$ and $I_{1}$ are modified Bessel functions of the first kind. For a plane sheet, the effectiveness factor is (Bird et al. 1960 problem 17.I)

$$
\eta_{p}(R, \lambda)=\left(\frac{\lambda}{R}\right) \tanh (R / \lambda)
$$

Again, these equations can be rewritten using the surface-to-volume ratio of sphere, cylinder, or plane sheet instead of their radii. In Fig. $2 b$ three graphs give the effectiveness factor as a function of $\lambda S / V$. 

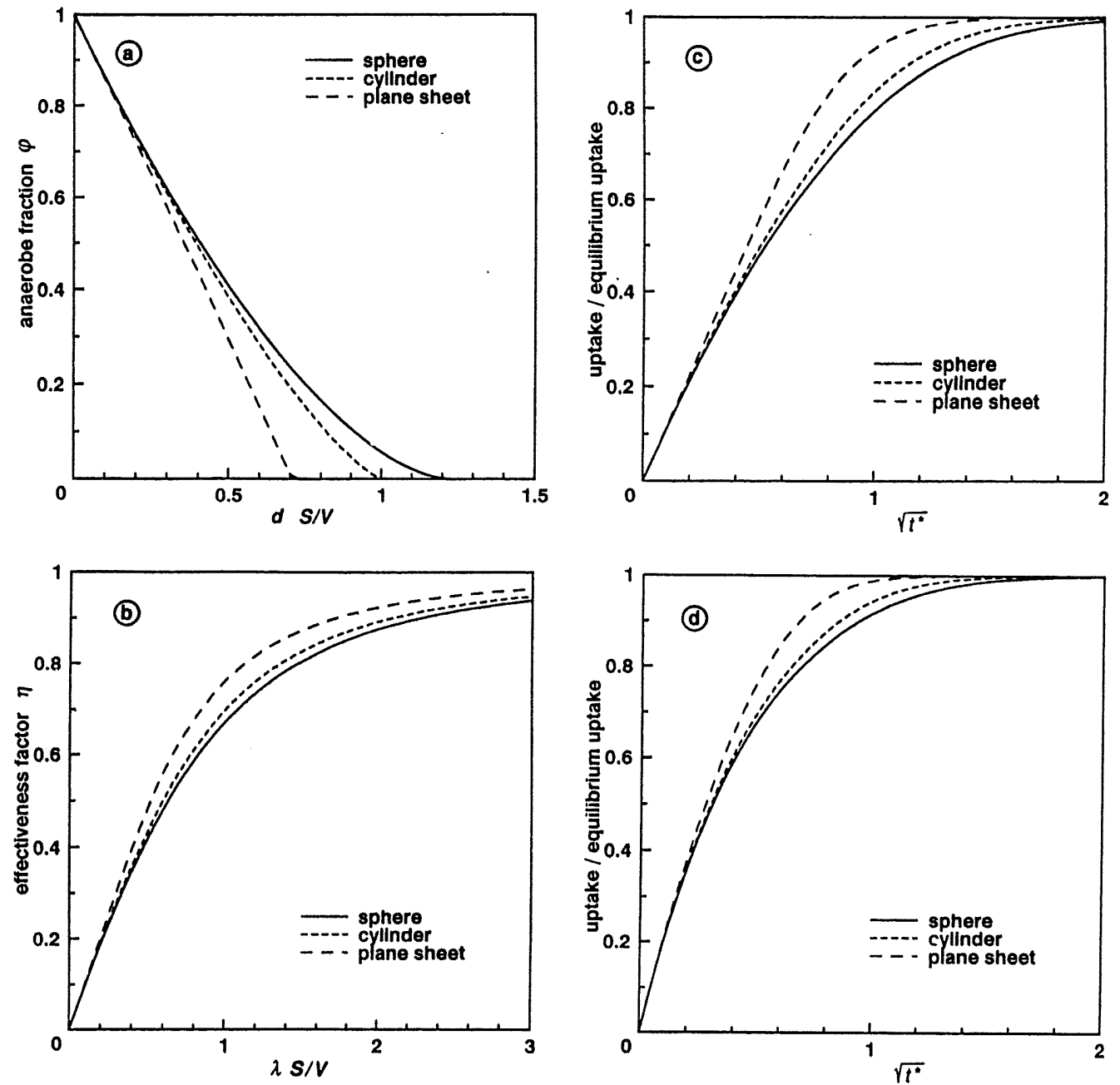

FIG. 2. Comparison of results of diffusion models applied to a sphere, a cylinder, and a plane sheet. Shape is of minor importance, provided aggregate size is expressed as a surface-to-volume ratio $S / V$. Different diffusion models have been used: (a) the steady state of oxygen diffusion with a zero-order respiration process; the diffusion distance $d$ is defined as $\sqrt{D C / Q}$ (cf. Eq. (1)); (b) the steady state of a first-order (oxygen) absorption process; $\eta$ is the aggregate absorption expressed as a fraction of the absorption for infinite $D$; the diffusion distance $\lambda$ is defined as $\sqrt{D / A}$ (cf. Eq. (5)); (c) gas or solute uptake after a sudden increase in outer concentration; after the increase at $t=0$, the outer concentration does not change; the dimensionless time $t^{*}$ is defined in Eq. (10); (d) after a sudden increase, the outer concentration slowly decreases due to the uptake of gas or solute by the aggregate; outer volume and aggregate volume have equal size.

\section{A sudden change in outer concentration}

When the concentration of a gas in the space around an aggregate suddenly changes, the concentration inside the aggregate will follow relatively slowly. It requires some time before a new equilibrium concentration is reached every- where. For simplicity, the description below refers to a sudden concentration rise from 0 to $C$. The results, however, are valid for a sudden change in general.

Two cases are distinguished. In the first case the outer concentration is supposed to-rise-suddenly at $t=0$ and remain constant after the 
change. It is not affected by the diffusive transport into the aggregate. In the second case the outer concentration also rises suddenly at $t=0$, but it decreases as a result of the subsequent flow of gas into the aggregate. The decrease depends on the surrounding volume. This volume is assumed to be $\alpha$ times as large as the aggregate volume $V$. For $\alpha=1$, for instance, aggregate volume and surrounding volume are equal, and the outer concentration will decrease until the new equilibrium concentration $C / 2$ is reached everywhere. For $\alpha=\infty$, the outer concentration does not decrease. Hence, the case of a constant outer concentration is a limiting case of the diffusion problem for a finite outer volume.

Crank (1956) refers to this problem as diffusion from a well-stirred solution of limited volume. It is thus assumed that no concentration gradient occurs outside the aggregate and that the initial rise in outer concentration is instantaneous. The differential equation for a sphere is

$$
4 \pi r^{2} \frac{\partial c}{\partial t}=\frac{\partial}{\partial r}\left(D 4 \pi r^{2} \frac{\partial c}{\partial r}\right)
$$

with the initial condition

$$
c(r, t)=0 \text { for } r<R, t=0
$$

and with boundary condition

$$
\frac{\partial c}{\partial t}=-\frac{3}{\alpha R} D \frac{\partial c}{\partial r} \text { for } r=R, t>0
$$

Crank (1956) gives an expression for the gas uptake $m_{s}(R, t)$ of the sphere. The uptake is expressed as a fraction of the uptake after infinite time (the equilibrium uptake). Expressions for the uptake $m_{c}(R, t)$ of a cylinder and $m_{p}(R, t)$ of a plane sheet can also be found in Crank (1956). Sphere, cylinder, and plane sheet radii can be replaced again by $3 \mathrm{~V} / \mathrm{S}, 2 \mathrm{~V} / \mathrm{S}$, and $V / S$, respectively. Then, in all three cases, the uptake becomes a function of a dimensionless time $t^{*}$ defined as

$$
t^{*}=t D(S / V)^{2}
$$

In Fig. 2c graphs are given for a constant outer concentration $(\alpha=\infty)$ and in Fig. 2d for equal aggregate and outer volume $(\alpha=1)$.

\section{Discussion of results for a single aggregate}

The curves in Fig. 2 for a sphere, a cylinder, and a plane sheet are close together. The result for an aggregate with another shape is likely to be somewhere in between. Therefore, for a real aggregate with an irregular shape, calculations need not be carried out. In a diffusion model the real aggregate can be represented by an equivalent sphere, cylinder, or plane sheet.

The use of an equivalent aggregate leads to approximate results. The differences in Fig. 2 between curves for a sphere, cylinder, and plane sheet give an impression of the size of the errors. For calculations on soil aggregates there seems to be no reason to require a higher accuracy. Other sources of error are likely to be at least as important.

Practical calculations require the use of a solubility and a soil porosity in the differential equation describing a process (e.g. Arah and Smith, 1989). The addition of these constants leads to a somewhat more complicated definition of the dimensionless groups used as $x$-coordinates in the graphs of Fig. 2. The graphs themselves remain unchanged, however, as does the conclusion with respect to the use of an $S / V$ ratio. Therefore, in this paper the differential equations have been used in their simplest form.

The surface-to-volume ratio $S / V$ of an aggregate may be regarded as a measure of its (inverse) size. For a constant aggregate shape, $S / V$ decreases with increasing aggregate size. Results of a diffusion model tend to depend on the size of an aggregate only, provided it is expressed as $S / V$. This formulation points towards the limitations of the $S / V$ method. Only a single number is used to measure aggregate size. When several length scales are involved, the method is likely to fail. In case of a rough or bumpy aggregate surface, for instance, there is a second length scale associated with the surface roughness. This problem can be solved by using the method for an aggregated soil described below. That method takes into account the presence of several length scales.

\section{METHOD FOR AN AGGREGATED SOIL}

\section{An analogy}

An approximate method is described for the application of a diffusion model to an aggregated soil. The method is necessarily more complicated than the method for a single aggregate discussed above. There is a close analogy, however, which is-expressed by the scheme-in Fig. 3. 
An aggregate of arbitrary shape with surface to volume ratio $S / V$

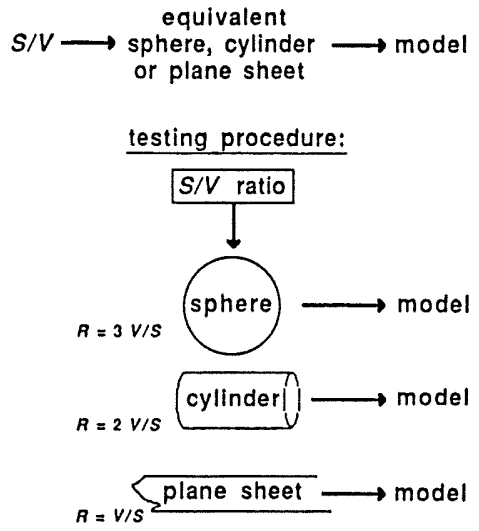

An aggregated soil with distance probability density $s(x)$

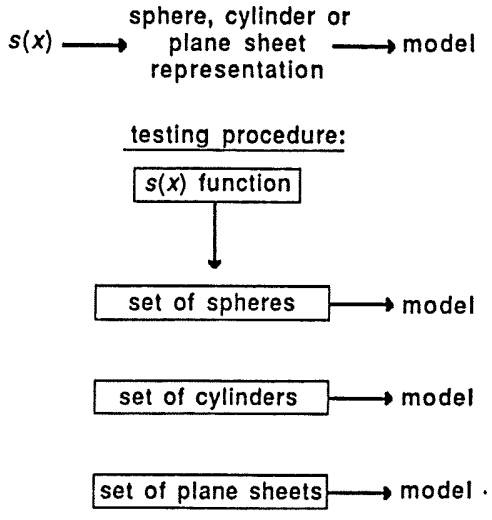

FIG. 3. The method for an aggregated soil is analogous to the use of an equivalent sphere, cylinder, or plane sheet for a single aggregate. Instead of a surface volume ratio $S / V$, a distance probability density function $s(x)$ is used. The two methods have been tested for several diffusion models (results in Figs. 2 and 8 ).

The left side of Fig. 3 recapitulates the method for a single aggregate. The geometry is characterized by a single number, the surface-to-volume ratio $S / V$. This number is used to calculate the size of an equivalent sphere, cylinder, or plane sheet (with equal $S / V$ ratio). In a diffusion model the real aggregate is represented by one of these equivalent aggregates. The method is tested by comparing diffusion model results for a sphere, a cylinder, and a plane sheet with equal $S / V$ ratios.

The right side of Fig. 3 shows a scheme of the method for an aggregated soil. Soil geometry is characterized by a distance probability density function $s(x)$, which is defined below. The function $s(x)$ plays the same role as the ratio $S / V$ in the method for a single aggregate. In case of a single aggregate, $S / V$ is used to define an equivalent aggregate. Similarly, a function $s(x)$ can be used to define a (hypothetical) model soil. A model soil is a set of spheres, cylinders, or plane sheets, which is characterized by a certain size distribution. This size distribution is chosen in such a way that model soil and real soil have equal functions $s(x)$. The model soil can be used then to represent the real soil in calculations on diffusion processes. The three types of model soil are therefore called the sphere, cylinder, and plane sheet representation of the real soil (see Fig. 3).

The use of a model soil in diffusion models is justified only when the result of a diffusion process is largely determined by the distance probability density function of the soil. Again, the influence of "shape" should be small. That has been verified by comparing results of diffusion models applied to a set of spheres, a set of cylinders, and a set of plane sheets with the same function $s(x)$.

In the first two sections (below) the distance probability density function $s(x)$ is introduced. Then the problem of finding a model soil with a given (i.e., measured) $s(x)$ is solved. Finally, the method is tested by examining the influence of shape.

The distance probability density function $s(x)$

Each point of the intra-aggregate space has a shortest distance $x$ to the inter-aggregate space. The distance $x$ belonging to a randomly chosen point can be considered as a stochastic variable. The function $s(x)$ is defined then as the probability density of $x$.

The definition of $s(x)$ as a distance probability density function is operational, i.e., it implies a way to measure $s(x)$ : for a large number of testpoints the shortest distance to the interaggregate space is measured. The testpoints may be either randomly chosen or may form a dense grid. $N$ distance intervals are chosen and the fraction of distances in each interval is calcu- 
lated. The result is a series of $N$ distance probabilities $p_{\mathrm{i}}$. When accurate distance probabilities are available for a large number of intervals, one might consider the estimation of a continuous $s(x)$ by means of a smoothing and interpolation technique.

Both a continuous function $s(x)$ and a series of distance probabilities can be used to derive a sphere, cylinder, or plane sheet representation of the real soil.

\section{The function $s(x)$ as a generalized surface-to- volume ratio}

Before we consider in detail the calculation of a model soil, the function $s(x)$ needs to be derived for an individual sphere, cylinder, and plane sheet. The definition of $s(x)$ as a distance probability density function can be directly applied. The distance $x$ is simply the distance from a randomly chosen point to the outer surface of the aggregate.

It is interesting, however, to consider an alternative definition of $s(x)$, which directly refers to the surfaces through which diffusive transports take place. The derivation below shows that the two definitions of $s(x)$ are equivalent for a cylinder.

For a long cylinder with radius $R$ and length $L$ the (inner) surface function $S(x)$ is defined as

$$
S(x)=2 \pi(R-x) L, \quad 0 \leq x \leq R
$$

It is the size of the surface at a distance $R-x$ from the center and at a distance $x$ from the outer surface. The integral of $S(x)$ over the interval $[0, R]$ is the total volume $\pi R^{2} L$ of the cylinder. Dividing $S(x)$ by this volume gives $s(x)$, the inner surface per unit volume

$$
s(x)=\frac{2}{R}\left(1-\frac{x}{R}\right), \quad 0 \leq x \leq R
$$

For $x=0, s(x)$ is equal to the ratio $S / V$ between outer surface and volume. The function $s(x)$ may thus be regarded as a generalized surface-tovolume ratio.

The integral of $s(x)$ over a distance interval $\left[x_{1}, x_{2}\right]$ is a volume fraction. It is the fraction of the aggregate volume between the inner surfaces at distances $x_{1}$ and $x_{2}$ from the outer surface. For a cylinder this is

$$
\int_{x_{1}}^{x_{2}} s(x) \mathrm{d} x=\left(1-\frac{x_{1}}{R}\right)^{2}-\left(1-\frac{x_{2}}{R}\right)^{2}
$$

In general a volume fraction can be treated as a probability. It is identical to the probability that a randomly chosen point is part of it. Hence, the above volume fraction is equal to the probability that the distance $x$, belonging to a randomly chosen point, lies in the interval $\left[x_{1}, x_{2}\right]$

$$
\operatorname{Pr}\left(x_{1}<x<x_{2}\right)=\int_{x_{1}}^{x_{2}} s(x) \mathrm{d} x
$$

This implies that $s(x)$, being an inner surface per unit volume, is also a distance probability density function. The two definitions are equivalent.

For a plane sheet, cylinder, and sphere, the desired function is most easily found as an inner surface per unit volume. The three functions are written as $s_{p}(R, x), s_{c}(R, x)$ and $s_{s}(R, x)$, respectively

$\begin{cases}s_{p}(R, x)=1 / R, & x \leq R \\ s_{p}(R, x)=0, & x>R\end{cases}$
$\begin{cases}s_{c}(R, x)=2 / R(1-x / R), & x \leq R \\ s_{c}(R, x)=0, & x>R\end{cases}$

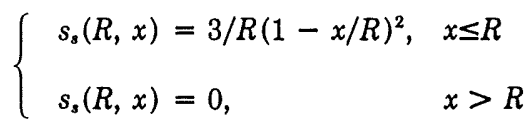

In Fig. 4 graphs have been drawn.

\section{$A$ model soil derived from a continuous $s(x)$}

First, an expression for the function $s(x)$ of a model soil must be derived making use of Eq. (12). Then the reverse problem of finding a model soil with a prescribed $s(x)$ can be solved.

A model soil is a set of flat, cylindrical, or spherical aggregates. The plane sheets, cylinders, or spheres do not possess a spatial position or an orientation. They are imaginary model aggregates, and their radii are their only properties. The model aggregates with radius in some interval $\left[R_{1}, R_{2}\right]$ occupy a fraction $\Delta v$ of the total model soil volume. This volume fraction $\Delta v$ is written as 


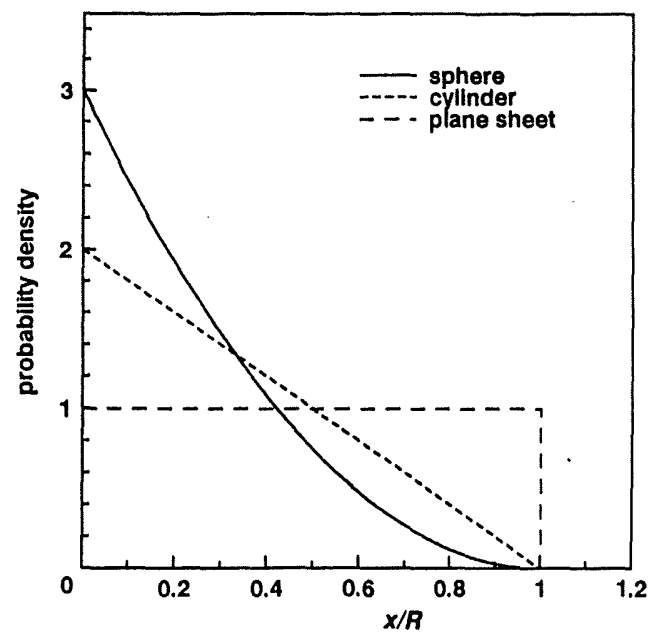

FIG. 4. The distance probability density function for a sphere, a cylinder, and a plane sheet (Eq. (12)).

The continuous function $v_{a}(R)$ is the yet unknown volume fraction density of the model soil (the subscript " $a$ " refers to "aggregate" which may be either "sphere," "cylinder," or "plane sheet," cf. Eqs. (6), (7), (8), and (12)). $v_{a}(R) \mathrm{d} R$ is the probability that a randomly chosen point belongs to a model aggregate with radius between $R$ and $R+\mathrm{d} R$. Then the $s(x)$ function of the model soil can be written as an integral over aggregate radius

$$
s(x)=\int_{x}^{\infty} s_{a}(R, x) v_{a}(R) \mathrm{d} R
$$

Here $s_{a}(R, x)$ is the distance probability density function of a model aggregate with radius $R(\mathrm{Eq}$ (12)). Eq. (13) shows how the $s(x)$ function of the model soil is "built up" from the distance probability density functions of spheres, cylinders, or plane sheets.

It is assumed now that a function $s(x)$ is known. This may be a theoretically calculated function of a measured function for a real soil. The distance probability density function of the model soil is set equal to this calculated or measured one. Then Eq. (13) becomes an equation with unknown $v_{a}(R)$. By solving this equation, a plane sheet, a cylinder, or a sphere model soil is found. In the Appendix it is shown that, for a plane sheet model soil

$$
v_{\bar{p}}(R)=-x \frac{\mathrm{d} s(x)}{\mathrm{d} x} / x=R
$$

for a cylinder model soil

$$
v_{c}(R)=\frac{1}{2} x^{2} \frac{\mathrm{d}^{2} s(x)}{\mathrm{d} x^{2}} / x=R
$$

for a sphere model soil

$$
v_{s}(R)=-\frac{1}{6} x^{3} \frac{\mathrm{d}^{3} s(x)}{\mathrm{d} x^{3}} / x=R
$$

Note that depending on the type of model soil, the calculation of $v_{a}(R)$ requires the existence of a first, second, or even third order derivative of $s(x)$.

As an example, the cylinder representation of a single sphere with radius $R_{s}$ is calculated. The distance probability density function for a sphere was given in Eq. (12b). After writing $R_{s}$ for the sphere radius, Eq. (14b) is applied. The volume fraction density as a function of the (cylinder) radius $R$ becomes

$$
v_{c}(R)=3 R^{2} / R_{s}{ }^{3}, \quad 0 \leq R \leq R,
$$

\section{A model soil derived from $N$ distance probabilities}

Instead of a continuous distance variable $x, N$ distance intervals are used. To each interval $i$ ( $i$ $=1, \ldots, N)$ belongs a distance probability $p_{i}$. That is the probability that the shortest distance $x$ from a randomly chosen point to the interaggregate space falls in the $i$-th interval.

The $i$-th distance interval is written as $\left[x_{i-1}\right.$, $x_{i}$ ]. The distance $x_{i}$ is the upper bound of the $i$ th interval and $x_{0}$ is defined to be zero. The probabilities $p_{i}$ can be measured by determining the distance $x$ for a large number of testpoints. Distance intervals are chosen and $p_{i}$ is estimated as the fraction of measured distances falling in interval $i$.

The $N$ distance probabilities $p_{i}$ can be used to define a (hypothetical) model soil. The model soil consists of plane sheets, cylinders, or spheres with $N$ different sizes. The $N$ aggregate radii are set equal to the upper bounds of the distance intervals. Hence

$$
R_{i}=x_{i}, \quad(i=1, \ldots, N)
$$

The aggregates with radius $R_{i}$ occupy a fraction $v_{i}$ of the model soil volume. The model soil is fully described by the $N$ fractions $v_{i}$, since the model soil aggregates do not possess a spatial position or orientation. The $N$ volume fractions $v_{i}$ need to be derived from the $N$ (measured) 
distance probabilities $p_{i}$. Two methods are given. The first method requires that all distance intervals have equal width. The probabilities $p_{i}$ will form a decreasing series (large distances are rare). If the $p_{i}$ are accurate numbers, one may estimate the derivatives in Eq. (14) from differences in successive $p_{i}$ values. For a plane sheet representation that leads to

$$
\left\{\begin{array}{l}
v_{i}=i\left(p_{i}-p_{i+1}\right), \quad i \leq N-1 \\
v_{N}=N p_{N}
\end{array}\right.
$$

for a cylinder representation

$\left\{\begin{array}{c}v_{i}=1 / 2 i^{2}\left(p_{i}-2 p_{i+1}+p_{i+2}\right), \\ i \leq N-2 \\ v_{i}=0, \quad i=N-1, N\end{array}\right.$

and for a sphere representation

$$
\left\{\begin{array}{c}
v_{i}=1 / 6 i^{3}\left(p_{i}-3 p_{i+1}+3 p_{i+2}-p_{i+3}\right) \\
i \leq N-3 \\
v_{i}=0, \quad i=N-2, N-1, N
\end{array}\right.
$$

In the Appendix it is shown that, for $N \rightarrow \infty$, these expressions correspond to Eq. (14) for a continuous $s(x)$ function. In fact, Eq. (17) is an attempt to approach a continuous solution without calculating a continuous $s(x)$. Satisfactory results require accurate probabilities $p_{i}$ and at least some tens of distance intervals.

The second method will be referred to as the matrix method. Volume fractions are calculated from the requirement that the model soil exactly reproduce the measured distance probabilities $p_{i}$. That requirement leads to a set of $N$ linear equations with $N$ unknown volume fractions

$$
p_{i}=\sum_{j=1}^{N} P_{i j} v_{j}, \quad i=1, \ldots, N
$$

The left side of each equation is a measured probability $p_{i}$. The right side is a distance probability calculated for the yet unknown model soil. The volume fraction $v_{j}$ is equal to the probability that a randomly chosen point lies within an aggregate with radius $R_{j}$. And $P_{i j}$ is defined as the probability that, given a point in an aggregate with radius $R_{j}$, the distance to the outer surface falls in the $i$-th distance interval. The probabilities (matrix elements) $P_{i j}$ are calculated from the-distance-probability density function for a plane sheet, cylinder, or sphere (see Eqs. (11) and (12)):

$$
P_{i j}=\int_{x_{i-1}}^{x_{i}} s_{a}\left(R_{j}, x\right) \mathrm{d} x
$$

The model soil volume fractions are found then by solving Eq. (18).

In principle, volume fractions can be calculated for an arbitrary number of distance intervals $N$. Different choices of $N$ lead to different model soils to which a diffusion model may be applied. In general, for a sufficiently large $N$, the result of a diffusion model becomes stable. A further increase of $N$ does not influence the result, which implies that the diffusion model converges. The choice of $N$ should be determined from that convergence. An example is given in the next section.

When all distance intervals have equal width, an expression can be derived for the matrix elements $P_{i j}$, and Eq. (18) can be solved analytically. Details are given in the Appendix. Here, the main results are described.

For plane sheets the solution of Eq. (18) again leads to Eq. (17a). Hence, in case of plane sheet volume fractions, the two methods coincide. Cylinder volume fractions derived with Eq. (18) tend to form an "oscillating" series. In a graph, the odd and even volume fractions would lie at opposite sides of a neat curve. In spite of oscillating volume fractions, however, the result of a diffusion model generally converges (see the next section for an example). The convergence even tends to be relatively fast, i.e., the matrix method requires fewer distance intervals than the use of Eq. (17b). That is an important advantage.

A sphere representation cannot be derived with the matrix method. Equation (18) leads to strongly oscillating sphere volume fractions, and results of diffusion models diverge for increasing $N$.

\section{Example}

Two cylinder representations of a single sphere with radius 1 have been calculated using different methods. To both cylinder representations a diffusion model describing local anaerobiosis has been applied. The two approximate results are compared with each other and with the exact result for the sphere.

The -first-cylinder-representation-considered is based on 20 distance probabilities $p_{i}(i=1$, 
$\ldots, 20)$. The $p_{i}$ have been calculated by integrating the distance probability density function of the sphere (Eq. 12c) over 20 distance intervals of equal width $\delta(=0.05)$. The matrix method has been used then to find the 20 cylinder volume fractions $v_{i}$ (Eq. (A3)). The volume fraction densities $v_{i} / \delta$ are given as a histogram in Fig. 5. Despite the fact that the used $p_{i}$ values are exact, the volume fractions oscillate strongly. It is shown below that these oscillations do not lead to problems in calculating anaerobe volume fractions for the cylinder representation.

The anaerobe volume fraction is calculated for the cylinder representation by applying the diffusion model to all cylinder size classes separately. Equation (4) describes the anaerobe fraction $\varphi_{\mathrm{c}}(R, d)$ of a cylinder with radius $R$. For each cylinder radius $R_{j}(j=1, \ldots, 20)$ this function is calculated and the results for the 20 cylinder size classes are added using the volume fractions $v_{j}$ as weights

$$
\varphi(d)=\sum_{j=1}^{N} v_{j} \varphi_{c}\left(R_{j}, d\right)
$$

In Fig. 6 a graph of this function has been drawn. It is close to the exact curve for the sphere which has been calculated with Eqs. (2) and (3).

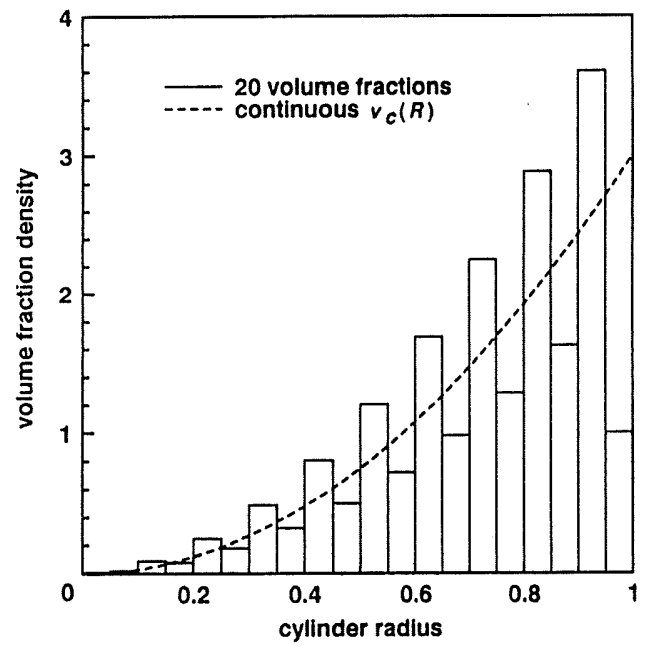

Fic. 5. Cylinder representations of a single sphere with $R_{a}=1$. The dashed line is the continuous volume fraction density derived as Eq. (15). The histogram consists of 20 volume fractions calculated with the matrix method (Eq. (A3) with $N=20$ ). The volume fractions have been divided by the class size 0.05 to get volume fraction densities.

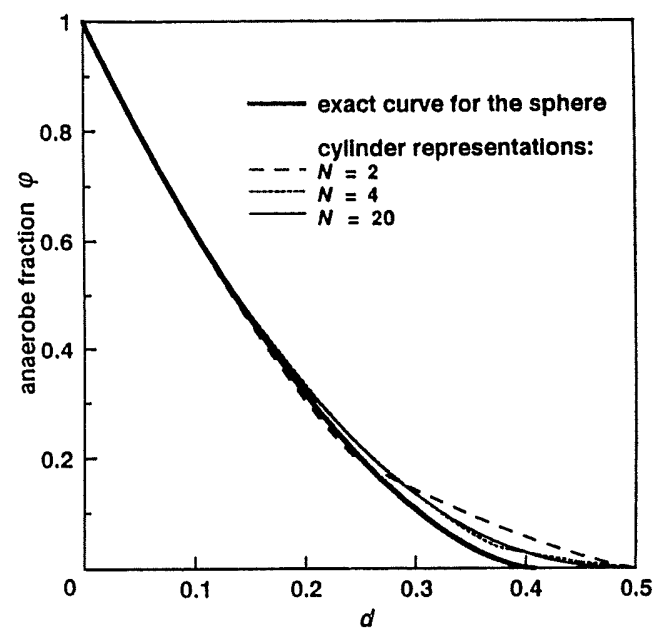

FIG. 6. The anaerobe volume fraction of a sphere with radius 1 (thick line). The other lines are obtained for cylinder representations derived with the matrix method. The results of the diffusion model converges for increasing $N$. The continuous cylinder representation in Fig. 5 also leads to the line for $N=20$.

It should be verified that the result for $N=$ 20 is insensitive to a change in the number of distance intervals $N$. Therefore the calculations have been repeated for $N=2$ and $N=4$ and the results have been drawn as dashed lines in Fig. 6 . The line for $N=4$ is already very close to the line for $N=20$. Hence, the result of the diffusion model indeed converges, and the limiting function is practically reached for $N=20$.

The second cylinder representation is directly based on the continuous distance probability density function of the sphere and was already derived above (Eq. (15)). A graph of $v_{c}(R)$ has been drawn in Fig. 5. For this cylinder representation the anaerobe volume fraction can be calculated by integrating $\varphi_{c}(R, d)$ over the cylinder volume distribution (instead of calculating a sum over $N$ size classes as in Eq. (20)). The result coincides with the line for $N=20$ in Fig. 6 , which implies that the two cylinder representations in Fig. 5 lead to the same diffusion model result.

\section{Testing the method}

The method for an aggregated soil can be tested by applying a diffusion model to a set of spheres, a set of cylinders, and a set of plane sheets with equal functions $s(x)$ (cf. Fig. 3). 
When the results for the three model soils are close together, the behavior of a real soil (with the same $s(x)$ ) is likely to be somewhere in between.

First, functions $s(x)$ have to be chosen from which model soils can be derived. The functions used have been calculated for a "sphere soil" consisting of spheres with a lognormal radius distribution. Lognormal aggregate size distributions have been observed in the field (Allmaras et al. 1965; Gardner 1956) and the large size ranges involved enable a thorough test of the method. Different values for the parameters of the lognormal distribution lead to different functions $s(x)$.

A lognormal radius distribution is characterized by a geometric mean radius $R_{g}$ and a factor $F$ expressing size variability. The radius interval of one standard deviation above as well as below the geometric mean is $\left[R_{g} / F, R_{g} F\right]$. When $F=$ 2 , for instance, $68 \%$ of the spheres have a radius between $R_{g} / 2$ and $2 R_{g}$. In the special case of $F$ $=1$ there is no size variability. Details can be found in the Appendix.

A certain choice of $R_{g}$ and $F$ corresponds to a certain volume fraction density function $v_{s}(R)$ of the sphere soil (see Appendix). The function $s(x)$ of the sphere soil is then found as (cf. Eq. (13))

$$
s(x)=\int_{x}^{\infty} s_{s}(R, x) v_{s}(R) \mathrm{d} R
$$

Since discrete probabilities are somewhat easier to handle than a continuous $s(x)$ function, distance probabilities $p_{i}$ have been calculated by integrating $s(x)$ over 50 distance intervals of equal length. Then Eq. (17) has been used to derive a set of spheres, a set of cylinders, and a set of plane sheets. Each of the three sets consists of 50 radius values with an associated volume fraction $v_{i}$.

As an example, graphs have been drawn in Fig. 7. The thick line is the continuous volume fraction density function $v_{s}(R)$ for the sphere soil with a lognormal radius distribution with parameters $R_{g}=1$ and $F=2$. From $s(x)$, calculated according to Eq. (21), three sets of volume fractions have been derived. As expected, the sphere representation (the nonconnected dots in Fig. 7) closely resembles the original sphere soil. The deviations result from the use of distinct size classes and from small numerical errors (cf.

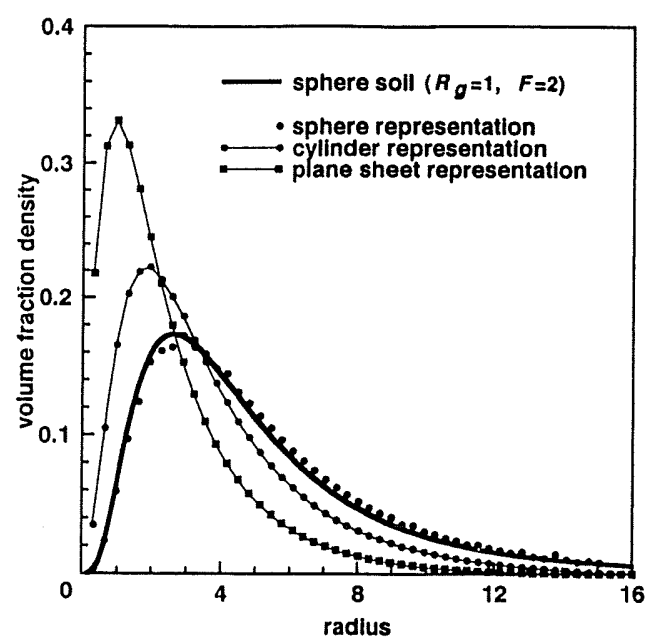

Fig. 7. Volume fraction density function for a "sphere soil" with a lognormal distribution of sphere radius (thick line). The points are volur.a fraction densities for a set of spheres, a set of cylinders, and a set of plane sheets representing the sphere soil (Eq. (17) with $N=50$ ). For clarity, cylinder and plane sheet volume fraction densities have been connected with a thin line.

the remark following Eq. (17)). The cylinder and plane sheet representation clearly have different volume fraction distributions.

A diffusion model is applied to a set of spheres, cylinders, or plane sheets by adding the results for the different radius values using the volume fractions $v_{i}$ as weights. For a first-order absorption process this is expressed by

$$
\eta(\lambda)=\sum_{i=1}^{N} v_{i} \eta_{a}\left(R_{i}, \lambda\right)
$$

$\eta_{a}(R, \lambda)$ is the effectiveness factor for a model aggregate (see Eqs. (6), (7), and (8)). The weighted sum $\eta(\lambda)$ is the effectiveness factor for the model soil. Other diffusion models can be applied by using similar expressions (cf. Eq. (20)).

\section{Results for an aggregated soil}

The method for an aggregated soil has been tested for the same diffusion models that have been used to test the method for a single aggregate (cf. Fig. 2). Anaerobe volume fractions have been calculated for three model soils with the same function $s(x)$. The calculations have been repeated for three different functions $s(x)$ be- 
longing to three values of $F$ (see previous section). Fig. 8 a shows the results. Indeed, for each choice of $F$, the curves for the three model soils are close together. Note that different choices of $R_{g}$ lead to the same graphs when $d / R_{g}$ is used as the independent variable.

In Fig. $8 \mathrm{~b}$ similar results are given for diffusion with a first-order absorption process.

In Fig. 8c graphs have been drawn of gas or solute uptake from the inter-aggregate space after a sudden rise in concentration at $t=0$.
After the sudden rise, the concentration in the inter-aggregate space is constant $(\alpha=\infty)$. The uptake of a model soil can then be calculated as a sum of analytic solutions $m_{a}\left(R_{i} t\right)$ with the volume fractions $v_{i}$ used as weights (see Eq. (22) and the explanation following Eq. (9)). The result is conveniently expressed as a function of a dimensionless time $t^{* *}$ defined as

$$
t^{* *}=t D / R_{g}^{2}
$$

When the concentration in the inter-aggre-
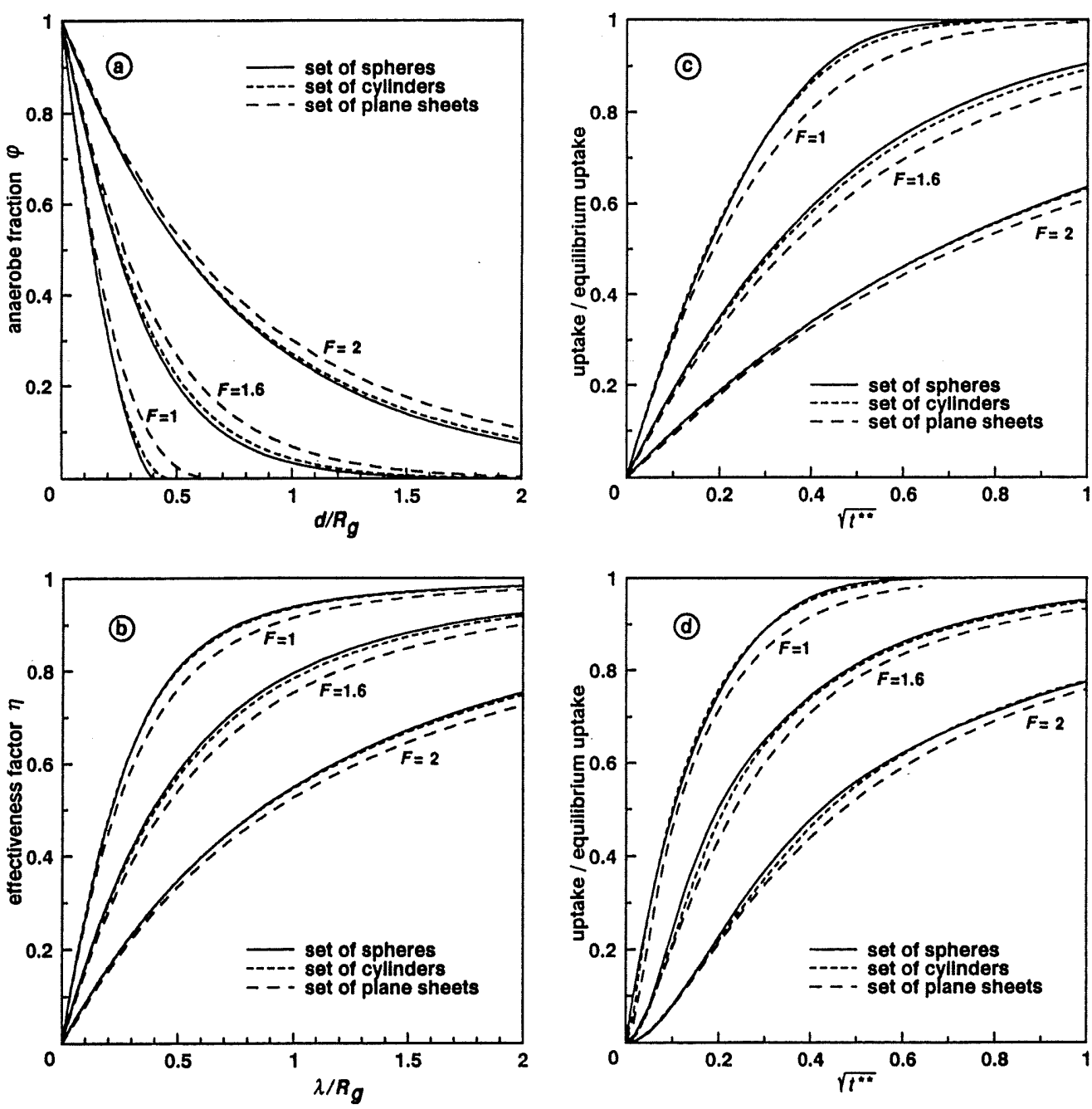

FiG. 8. Comparison of results of diffusion models applied to a set of spheres, a set of cylinders, and a set of plane sheets with equal $s(x)$ functions (cf. Fig. 2). Each set of three lines corresponds to a different choice for $s(x)$ (see Eq. (21) and Appendix). The same four models have been used as in Fig. 1. The dimensionless time $t^{* *}$ used in (c) and (d) is defined in Eq. (23). 
gate space decreases as a result of the uptake by the soil, the processes in small and large aggregates will influence each other. Small aggregates quickly approach equilibrium with the surrounding concentration. Then, due to the absorption by the larger aggregates, the inter-aggregate concentration decreases, and the gas or solute content of the smaller aggregates may even start to decrease. Clearly this cannot be described by making use of analytic solutions for a single aggregate. The total uptake has to be found by numerical simulation of the uptake for all aggregate size classes. Fig. $8 d$ shows the results. The volume of the inter-aggregate space was set equal to the total aggregate volume $(\alpha=1)$. Again the curves for spheres, cylinders, and plane sheets are close together. (For short times, numerical errors cause the calculated uptakes to be somewhat too small; initial uptake should be proportional to the square root of time.)

\section{Numerical aspects}

The calculations discussed in this paper have been carried out by means of about 70 commented (sub)programs written in standard FORTRAN-77. They are available on request.

The distance probability density fucntions $s_{a}(R, x)$ and the solutions of the used diffusion models $\varphi_{a}(R, d), \eta_{a}(R, \lambda)$, and $m_{a}(R, t)$ have been written as FORTRAN functions. This considerably simplifies the subsequent calculation of integrals or weighted sums over aggregate radius (cf. Eqs. (20), (21), and (22)). Integrals have been calculated by means of the GaussLegendre method or by using the Simpson rule in a form enabling accuracy control (Press et al. 1986). The results in Fig. $8 \mathrm{~d}$ have been calculated by simulating concentrations using a fourth order Runge Kutta algorithm with stepsize control (Press et al. 1986).

\section{ADAPTATION TO WORMHOLES AND ROOTS}

When diffusive transports are considered to or from (worm)holes, the method described above requires some adaptation. All holes together form the inter-aggregate space, and the distance $x$ belonging to a point of the soil is defined as the distance to the center of the nearest hole. The distance probability density function $s(x)$ will increase for short distances according to the size of concentric surfaces around the holes. For large distances, $s(x)$ de- creases and eventually reaches zero. A model soil can be derived from such a function.

Cylinders with a hole along their axes are used as model "aggregates." Diffusive transports in the model soil only take place through the surface of the hole. The outer cylinder surface is subject to a zero-flow condition. In this way the shape of the model soil is somewhat adapted to the system to be described. Again, the cylinder radius values are set equal to the upper bounds of $N$ distance intervals. In the Appendix it is shown that the cylinder volume fractions are then given by

$$
\left\{\begin{array}{l}
v_{i}=i^{2}\left(\frac{p_{i}}{2_{i-1}}-\frac{p_{i+1}}{2 i+1}\right), \quad i<N \\
v_{N}=\frac{N^{2}}{2 N-1} p_{N}
\end{array}\right.
$$

The cylinder volume fractions are independent of the hole diameters since $x$ is defined as the distance to the center of the nearest hole. In model calculations the diameter of the holes along the cylinder axes may be chosen according to a measured or assumed size distribution.

This method may also be used for the calculation of solute uptake by a root system. The model soil then consists of cylinders with a root along their axes. The different cylinder size classes will correctly represent the soil volume fractions at different distances from the root system.

\section{CONCLUSIONS}

For a single aggregate and for an aggregated soil, shape has a minor influence on results of diffusion models (Figs. 2 and 8). That provides the use of a simple model soil with a physical basis. The use of a model soil is not restricted to demonstrative, theoretical calculations. Despite its geometrical simplicity, a model soil can be used in quantitative calculations on diffusion processes. Moreover, a complicated and realistic description of the geometry of an aggregated soil would probably lead to marginal improvements only, at the expense of computational and conceptual simplicity.

Different types of transport models may make use of a model soil. The only requirement is that model results are relatively independent of shape. Models on gas diffusion and diffusion of solute in water are the most obvious examples. For heat transport the method will also work 
since diffusion and heat transport are very similar processes. Also gravity-free flow of water in soil aggregates can be formulated as diffusion. In case of doubt one may test the shape dependence of a transport model by applying it to (sets of) spheres, cylinders, and plates.

Soil aggregates share their "outer world," the inter-aggregate space. If the condition of the inter-aggregate space is influenced by diffusive flows into or from aggregates, the aggregates of a model soil cannot be considered as independent objects. As a consequence, the application of a diffusion model requires numerical simulation of concentrations even if the diffusion model is a simple one (e.g. Fig. 8d). An elaborate example would be the simulation of water vapor pressure in cracks, influenced by exchange with the atmosphere and by transport of water and water vapor in a cylinder model soil.

The sphere, cylinder, or plane sheet volume fractions of a model soil are calculated from distance probabilities. Sphere volume fractions are very sensitive to errors and can only be calculated when at least some tens of accurate distance probabilities are available. Most appropriate in many situations will be the calculation of cylinder volume fractions with the matrix method. One should try a few values $N$, the number of distance intervals, in order to verify the convergence of diffusion model results. Note that distance intervals need not have equal widths (see Appendix). Plane sheet volume fractions are easy to calculate and may be used if cylinder volume fractions cannot be obtained.

Distance probabilities can be derived from measured distances. In principle, spatial distances are needed, measured in a three-dimensional image of the soil. In a few cases simplifications are possible. When cracks are vertical, for instance, spatial distances can be determined in a horizontal intersection. Also, in case of an isotropic soil structure, one may use distances measured in the plane of an intersection. That requires an adaptation of the method for calculating model soil volume fractions. In a subsequent paper this will be discussed in detail.

The method developed in this paper requires the existence of a distinction between a relatively uniform porous medium and an interaggregate space around it. Such a distinction cannot be made if there is a continuous size distribution of macropores and micropores. The soil-cannot-be regarded then-as-an-aggregated system, and the method described in this paper will fail.

A distance probability distribution may also be useful, independent of physical transport models. When studying displacements of animals or bacteria, for instance, one may wish to calculate the soil volume fraction at a given distance from the inter-aggregate space; a distance probability density function is exactly what is needed.

\section{APPENDIX}

\section{$A$ continuous $s(x)$}

The problem of finding a model soil volume fraction density $v_{a}(R)$ from a function $s(x)$ was formulated in Eq. (13) as

$$
s(x)=\int_{x}^{\infty} s_{a}(R, x) v_{a}(R) \mathrm{d} R
$$

Here $s(x)$ is a prescribed distance probability density function (p.d.f.) and $s_{a}(R, x)$ is the distance p.d.f. of a sphere, cylinder, or plane sheet. The lower bound of the integration interval can be set to $x$ since the functions $s_{a}(R, x)$ are zero for $R<x$ (see Eq. (12)). Differentiating both sides of Eq. (A1) leads to

$$
\begin{aligned}
& \frac{\mathrm{d} s(x)}{\mathrm{d} x}=-s_{a}(R, x) v_{a}(R) / /_{R=x} \\
& +\int_{x}^{\infty} \frac{\mathrm{d} s_{a}(R, x)}{\mathrm{d} x} v_{a}(R) \mathrm{d} R
\end{aligned}
$$

For a plane sheet the integrand in this expression is zero. Hence, for a plane sheet model soil

$$
\frac{\mathrm{d} s(x)}{\mathrm{d} x}=-\frac{1}{x} v_{\rho}(x)
$$

which is equivalent to Eq. (14a). The solutions for a cylinder and a sphere representation are found by differentiating Eq. (A1) two and three times, respectively.

\section{Approximation of a continuous solution with $N$ probabilities $p_{i}$}

Instead of a continuous distance p.d.f. $s(x)$, only $N$ distance probabilities $p_{i}$ are known for $N$ intervals $\left[x_{i-1}, x_{i}\right]$ with $x_{0}=0$ and $x_{N}$ the largest distance found. The $p_{i}$ are related to an underlying $s(x)$ function according to

$$
p_{i}=\int_{x_{i-1}}^{x_{i}} s(x) \mathrm{d} x
$$


If all distance intervals have equal width $\delta$, differences between successive $p_{i}$ values reflect differences in the value of $s(x)$. The forward difference operator $\Delta$ is used for a short notation

$$
\begin{gathered}
\Delta p_{i}=p_{i+1}-p_{i} \\
\Delta^{2} p_{i}=p_{i+2}-2 p_{i+1}+p_{i} \\
\Delta^{3} p_{i}=p_{i+3}-3 p_{i+2}+3 p_{i+1}-p_{i}
\end{gathered}
$$

The $N$ aggregate size classes of the model soil are chosen according to Eq. (16). The associated volume fractions $v_{i}$ have to be found from the distance probabilities $p_{i}$. Using the notation above, the finite difference version of Eq. (14) becomes

for plane sheets

$$
v_{i}=-i \Delta p_{i}
$$

for cylinders

$$
v_{i}=1 / 2 i^{2} \Delta^{2} p_{i}
$$

for spheres

$$
v_{i}=-1 / 6 i^{3} \Delta^{3} p_{i}
$$

To prove these relations we show that for a fixed $x$, the volume fraction density $v_{i} / \delta$ approaches the continuous $v(x)$ derived above. The proof makes use of Taylor expansions of $s(x)$. In case of plane sheets the definition of the first order derivative is equivalent to

$$
s(x+\delta)-s(x)=\delta \frac{\mathrm{d} s(x)}{\mathrm{d} x}, \quad(\delta \downarrow 0)
$$

Multiplying this by $\delta$ gives

$$
p_{i+1}-p_{i}=\delta^{2} \frac{\mathrm{d} s(x)}{\mathrm{d} x}, \quad(\delta \downarrow 0)
$$

And, using $i=x / \delta$ for a fixed value of $x$

$$
\lim _{\delta \downarrow 0}\left(v_{i} / \delta\right)=\lim _{\delta \downarrow 0}\left(-i \Delta p_{i} / \delta\right)=-x \frac{\mathrm{d} s(x)}{\mathrm{d} x}
$$

With higher order Taylor expansions the other expressions in Eq. (A2) can be proven in the same way. The choice of $v_{N}$ in Eq. (17a) is motivated by the result of the matrix method for plane sheets but may well be understood by assuming $p_{N+1}=0$.

\section{Matrix method}

The $N$ distance intervals need not have equal width. The aggregate size classes of the model soil are defined again by Eq. (16). The corresponding volume fractions are found from the requirement that distance probabilities in soil and model soil are precisely equal. That leads to a set of $N$ equations with $N$ unknown volume fractions $v_{j}$ (Eq. (18)). The matrix elements $P_{i j}$ are calculated with Eq. (19). The volume fractions are found then by inverting the matrix $P$ after calculating all its elements. Note that the distance probability density function $s_{a}\left(R_{j}, x\right)$ in Eq. (19) is an arbitrary function in principle. A model soil does not necessarily consist of plane sheets, cylinders, or spheres.

For $N$ distance intervals of equal width, analytic solutions for a plane sheet, cylinder, and sphere model soil are given below.

\section{Solution for plane sheets}

Matrix elements $P_{i j}(i, j=1, \ldots, N)$ are calculated with Eqs. (12a) and (19).

$$
\begin{cases}P_{i j}=\frac{1}{j}, & i \leq j \\ P_{i j}=0, & i>j\end{cases}
$$

The inverse of the matrix $P$ is given by

$$
\begin{cases}P_{i j}^{-1}=0, & j<i \text { or } j>i+1 \\ P_{i j}^{-1}=i, & j=i \\ P^{-1}=-i, & j=i+1\end{cases}
$$

This can be shown by calculating $P^{-1} P$. The plane sheet volume fractions following from this inverse matrix are exactly the ones given in Eq. (17a).

\section{Solutions for cylinders}

Matrix elements $P_{i j}(i, j=1 \ldots N)$ are calculated with Eqs. (12b) and (19). In case of $N$ equal distance interval

$$
\begin{cases}P_{i j}=\frac{2(j-i)+1}{j^{2}}, & i \leq j \\ P_{i j}=0, & i>j\end{cases}
$$

The inverse $P^{-1}$ is given by

$$
\begin{cases}P_{i j}^{-1}=0, & j<i \\ P_{i j}^{-1}=i^{2}, & j=i \\ P_{i j}^{-1}=-3 i^{2}, & j=i+1 \\ P_{i j}^{-1}=(-1)^{j-i} 4 i^{2}, & j \geq i+2\end{cases}
$$

Again this can be proven by carefully calculating the elements of $P^{-1} P$. The volume fractions 


$$
\begin{aligned}
& \text { wing from } P^{-1} \text { are } \\
& \left\{\begin{array}{lr}
v_{i}=i^{2}\left[p_{i}-3 p_{i+1}+4 \sum_{j=i+2}^{N}(-1)^{j-i} p_{j}\right], \\
v_{i}=i^{2}\left(p_{i}-3 p_{i+1}\right), & i \leq N-2 \\
& i=N-1 \\
\mathrm{v}_{N}=\mathrm{N}^{2} \mathrm{p}_{N} &
\end{array}\right.
\end{aligned}
$$

The expression for $i \leq N-2$ is not equal to Eq. (A2b). The volume fraction densities $v_{i} / \delta$ do not necessarily converge to the continuous $v(R)$ for large $N$. From Eq. (A3), however, it follows that

$$
\frac{1}{2}\left[\frac{v_{i}}{i^{2}}+\frac{v_{i+1}}{(i+1)^{2}}\right]=\frac{1}{2} \Delta^{2} p_{i}, \quad i \leq N-2
$$

This implies that the average of two successive volume fractions approaches the desired limit for $N \rightarrow \infty$. Individual volume fractions, however, may "oscillate": odd and even elements of the series $v_{i} / \delta$ lie at opposite sides of a continuous $v(R)$. Such instabilities can be easily produced by errors in the probabilities $p_{i}$ (the sign of a certain $p_{i}$ in Eq. (A3) is different in $v_{i}$ and $\left.v_{i+1}\right)$. Oscillations may also have a purely mathematical background, for instance in the case of the cylinder representation of a single sphere (Fig. 5).

Oscillations between successive volume fractions only lead to unstable diffusion model results if they are severe. If the solution of the applied diffusion model is continuous in the cylinder radius, the oscillations will (partly) outweigh each other. The matrix method has the advantage over Eq. (17b) that the model soil exactly reproduces the distance probabilities $p_{i}$. This probably causes diffusion model results to converge for relatively low values of $N$. The lines in Fig. 8 for cylinders were calculated using Eq. (17b) with $N=50$. Using the matrix method, 20 size classes are sufficient (see also the convergence of the lines in Fig. 6 for increasing $N$ ).

Note that Eq. (A3) may lead to negative volume fractions. That will be the case when the $p$ values form a slowly decreasing or even an increasing series. Negative volume fractions are not a problem in principle. As long as the applied diffusion model converges for increasing $N$, the method works. Increasing distance probabilities, however, imply that the intra-aggregate space (partly) consists of holes. Another type of model soil might then lead to better results (see Adaptation to Wormholes and Roots).

\section{Solution for spheres}

Matrix elements $P_{i j}(i, j=1, \ldots, N)$ are calculated with Eqs. (12c) and (19). In case of $N$ equal distance intervals

$$
\begin{cases}P_{i j}=\frac{3(j-i)^{2}+3(j-i)+1}{j^{3}}, & i \leq j \\ P_{i j}=0, & i>j\end{cases}
$$

The inverse for $N=6$ is

$$
\left(\begin{array}{rrrrrr}
1 & -7 & 30 & -114 & 426 & -1590 \\
0 & 8 & -56 & 240 & -912 & 3408 \\
0 & 0 & 27 & -189 & 810 & -3078 \\
0 & 0 & 0 & 64 & -448 & 1920 \\
0 & 0 & 0 & 0 & 125 & -875 \\
0 & 0 & 0 & 0 & 0 & 216
\end{array}\right)
$$

Sphere volume fractions calculated from this inverse matrix will generally oscillate between large negative and positive values. For increasing $N$, the oscillations increase. This leads to diverging results when a diffusion model is applied to a sphere representation. Hence, sphere volume fractions should not be calculated with the matrix method.

\section{Cylinders with a hole or root along the axis}

When the inter-aggregate space consists of (worm)holes or in the case of a root system, the geometry of the model soil may be adapted in order to prevent errors from becoming too large. Again, a model soil may be derived consisting of cylinders with various sizes. The inter-aggregate space is situated along the axes of the cylinders in the form of a hole or root. The outer-cylinder surface is subject to a zero flow condition. The hole or root diameters can be taken equal to measured ones. Cylinder volume fractions are calculated again from a distance probability density function $s(x)$. For each point, $x$ is defined as the distance from that point to the center of the nearest hole (or root). Hence, the distance p.d.f. of the soil is independent of the size of the holes. The calculation of cylinder volume fractions is based on the distance p.d.f. $s(R, x)$ for a single cylinder

$$
\begin{cases}s(R, x)=2 x / R^{2}, & x \leq R \\ s(R, x)=0, & x>R\end{cases}
$$


Again, $N$ distance intervals are chosen with upper bounds $x_{i}(i=1, \ldots, N)$. For equal interval widths, Eqs. (16) and (19) lead to

$$
\begin{cases}P_{i j}=\frac{2 j-1}{j^{2}}, & i \leq j \\ P_{i j}=0, & i>j\end{cases}
$$

with inverse

$$
\begin{cases}P_{i j}^{-1}=\frac{i^{2}}{2 i-1}, & j=i \\ P_{i j}^{-1}=\frac{-i^{2}}{2 i+1}, & j=i+1 \\ P_{i j}^{-1}=0, & j<i \text { or } j>i+1\end{cases}
$$

From this expression Eq. (24) follows directly for the cylinder volume fractions.

\section{A lognormal sphere radius distribution}

The $s(x)$ functions used for testing the method for an aggregated soil were calculated for a "sphere soil" with a lognormal radius distribution. The probability density function $p(R)$ is

$$
p(R)=\frac{1}{R} \frac{1}{\sigma \sqrt{2 \pi}} \exp \left\{-\frac{(\ln R-\mu)^{2}}{2 \sigma^{2}}\right\}
$$

Here $\mu$ and $\sigma$ are the mean and standard deviation of the normal distribution (of $\ln R$ ), respectively. The geometric mean radius $R_{g}$ and the variability factor $F$ are defined as

$$
R_{g}=\mathrm{e}^{\mu} \text { and } F=\mathrm{e}^{\sigma}
$$

Using these relations, $p(R)$ may be calculated for any choice of $R_{g}$ and $F$. The average sphere volume $\bar{V}$ is found as

$$
\nabla=\int_{0}^{\infty} \frac{4}{3} \pi R^{3} p(R) \mathrm{d} R
$$

The volume fraction density $v_{0}(R)$ is then given by

$$
v_{\triangleleft}(R)=\frac{1}{\bar{V}} \frac{4}{3} \pi R^{3} p(R)
$$

The $s(x)$ function belonging to a certain choice of $R_{g}$ and $F$ is calculated according to Eq. (21). Note that in case of a lognormal radius distribution aggregate volume is also lognormally distributed. The variability factor belonging to that distribution is equal to $F^{3}$.

\section{ACKNOWLEDGMENTS}

I thank C. A. van Diepen, J. Goudriaan, M. J. Kropff, P. A. Leffelaar, P. A. C. Raats, J. H. G. Verhagen, and especially C. T. de Wit for discussions on the subject, their comments and their questions. I also wish to thank an anonymous reviewer for his (or her) many helpful remarks.

\section{REFERENCES}

Allmaras, R. R., R. E. Burwell, W. B. Voorhees, and W. E. Larson. 1965. Aggregate size distribution in the row zone of tillage experiments. Soil Sci. Soc. Am. Proc. 29:645-650.

Arah, J. R. M., and K. A. Smith. 1989. Steady-state denitrification in aggregated soils. A mathematical model. J. Soil Sci. 40:139-149.

Bird, R. B., W. E. Steward, and E. N. Lightfood. 1960 Transport phenomena. Wiley \& Sons, New York.

Crank, J. 1956. The mathematics of diffusion. Clarendon Press, Oxford.

Currie, J. A. 1961. Gaseous diffusion in aeration of aggregated soils. Soil Sci. 92:40-45.

Gardner, W. R. 1956. Representatiun of soil aggregatesize distribution by a logarithmic-normal distribution. Soil Sci. Soc. Am. Proc. 20:151-153.

Greenwood, D. J. 1961. The effect of oxygen concentration on the decomposition of organic materials in soil. Plant Soil 14:360-376.

Leffelaar, P. A. 1987. Dynamics of partial anaerobiosis, denitrification, and water in a soil aggregate: Simulation. Soil Sci. 146:427-444.

Press, W. H., B. P. Flannery, S. A. Teukolsky, and W. T. Vetterling. 1986. Numerical recipes, the art of scienctific computing. Cambridge University Press, New York.

Smith, K. A. 1977. Soil aeration. Soil Sci. 123:284291. 